\title{
Preorders in Simple Games
}

\author{
Josep Freixas and Montserrat Pons \\ Universitat Politècnica de Catalunya, Department of Mathematics, Manresa, Spain, \\ josep.freixas@upc.edu, \\ montserrat.pons@upc.edu
}

\begin{abstract}
Any power index defines a total preorder in a simple game and, thus, induces a hierarchy among its players. The desirability relation, which is also a preorder, induces the same hierarchy as the Banzhaf and the Shapley indices on linear games, i.e., games in which the desirability relation is total. The desirability relation is a sub-preorder of another preorder, the weak desirability relation, and the class of weakly linear games, i.e., games for which the weak desirability relation is total, is larger than the class of linear games. The weak desirability relation induces the same hierarchy as the Banzhaf and the Shapley indices on weakly linear games. In this paper, we define a chain of preorders between the desirability and the weak desirability preorders. From them we obtain new classes of totally preordered games between linear and weakly linear games.
\end{abstract}

Keywords: Simple game, Power index, Preorder, Desirability, Weak desirability, Linear game, Weakly linear game

\section{Introduction}

Any power index considered in a simple game induces a total preorder on the set of players, and, thus, a hierarchy among them. Two power indices which induce the same hierarchy in a simple game are said to be ordinally equivalent in it. In this paper we refer to three power indices: the Shapley-Shubik index (SS, henceforth) $[14,15]$, the Penrose-Banzhaf-Coleman index (PBC, henceforth) [12, $1,4]$ and the Johnston index [11]. It is known $[5,13]$ that the PBC, the SS and the Johnston power indices are ordinally equivalent in linear games, and that the common induced hierarchy is the one given by the desirability relation.

In [3] weakly linear games were introduced and it was proved that all regular semivalues (i.e., semivalues with positive coefficients for the marginal contributions, see $[2,3]$ ) are ordinally equivalent for this kind of games, and that the common induced hierarchy is the one given by the weak desirability relation. As every linear simple games is weakly linear, and both the PBC and the SS power indices are regular semivalues, this work extends and generalizes the former ones in relation with these two indices. The ordinal equivalence of the SS, the PBC and the Johnston indices in a class larger than linear games but smaller than weakly linear games was proved in [7]. 
In this paper, a chain of families of simple games, between linear and weakly linear games, are defined and some of their properties are studied. In all these classes of simple games, the Banzhaf and the Shapley-Shubik indices are ordinally equivalent. The smallest family in this chain is the class of linear games and the largest one is the class of weakly linear games. A somehow similar work was developed in [16] by defining a chain of classes of simple games between weighted simple games and linear simple games.

The paper is organized as follows. Basic definitions and preliminary results are included in Section 2. Section 3 contains new characterizations of the desirability and the weak desirability relations. In Section 4 the $m$-desirability relations are defined and some of their properties are studied. Some Conclusions end the paper in Section 5.

\section{Definitions and preliminaries}

In the sequel, $N=\{1,2, \ldots, n\}$ denote a fixed but otherwise arbitrary finite set of players. Any subset $S \subseteq N$ is a coalition. A simple game $v$ (in $N$, omitted hereafter) is a cooperative game, i.e., a function $v: 2^{N} \rightarrow \mathbb{R}$ with $v(\emptyset)=0$, such that: (a) $v(S)=0$ or 1 for any coalition $S,{ }^{1}$ (b) $v$ is monotonic, i.e., $v(S) \leq v(T)$ whenever $S \subset T$, and (c) $v(N)=1$. Either the family of winning coalitions $\mathcal{W}=\mathcal{W}(v)=\{S \subseteq N: v(S)=1\}$ or the subfamily of minimal winning coalitions $\mathcal{W}^{m}=\mathcal{W}^{m}(v)=\{S \in \mathcal{W}: T \subset S \Rightarrow T \notin \mathcal{W}\}$ determines a simple game.

Given a simple game $v$, let us consider, for each $i \in N$, and for every integer $h$ with $1 \leq h \leq n$, some important subsets of $N$ :

$$
\mathcal{P}_{i}=\{S \subseteq N: i \in S\} \quad \text { and } \quad \mathcal{P}_{i}(h)=\left\{S \in \mathcal{P}_{i}:|S|=h\right\} .
$$

$\mathcal{P}_{i}$ is the set of coalitions $S$ that contain $i$, while $\mathcal{P}_{i}(h)$ is the subset of such coalitions having cardinality $h$.

$$
\mathcal{W}_{i}=\{S \in \mathcal{W}: i \in S\} \quad \text { and } \quad \mathcal{W}_{i}(h)=\left\{S \in \mathcal{W}_{i}:|S|=h\right\} .
$$

$\mathcal{W}_{i}$ is the set of winning coalitions $S$ that contain $i$, while $\mathcal{W}_{i}(h)$ is the subset of such coalitions having cardinality $h$.

$$
\mathcal{C}_{i}=\left\{S \in \mathcal{W}_{i}: S \backslash\{i\} \notin \mathcal{W}\right\} \quad \text { and } \quad \mathcal{C}_{i}(h)=\left\{S \in \mathcal{C}_{i}:|S|=h\right\} .
$$

$\mathcal{C}_{i}$ is the set of winning coalitions $S$ that are crucial for $i$, while $\mathcal{C}_{i}(h)$ is the subset of such coalitions having cardinality $h$. It is obvious that

$$
\mathcal{C}_{i}(h) \subseteq \mathcal{W}_{i}(h) \subseteq \mathcal{P}_{i}(h)
$$

Notice that for $h=1$ the set $\mathcal{P}_{i}(1)$ only contains the singleton $\{i\}, \mathcal{W}_{i}(1)=$ $\mathcal{C}_{i}(1)$ and $\{i\} \in \mathcal{C}_{i}(1) \Leftrightarrow\{i\} \in \mathcal{W}$. On the other extreme, for $h=n$ the set $\mathcal{P}_{i}(n)$ only contains the total set $N, \mathcal{W}_{i}(n)=\mathcal{P}_{i}(n)$ and $N \in \mathcal{C}_{i}(n) \Leftrightarrow N \backslash\{i\} \notin \mathcal{W}$.

\footnotetext{
${ }^{1}$ For a detailed discussion of some issues raised by allowing abstentions, see Felsenthal and Machover [6] and for several levels of approval in input and output, see Freixas and Zwicker [9].
} 
Definition 1. The desirability relation ([10])

Let $v$ be a simple game and $i, j \in N$. Then

$$
\begin{aligned}
& i \succsim_{D} j \quad \Leftrightarrow \quad\{S \cup\{j\} \in \mathcal{W} \Rightarrow S \cup\{i\} \in \mathcal{W}\} \text { for any } S \subseteq N \backslash\{i, j\}, \\
& i \succ_{D} j \quad \Leftrightarrow \quad i \succsim_{D} j \text { and } j \succsim_{D} i, \\
& i \approx_{D} j \quad \Leftrightarrow \quad i \succsim_{D} j \text { and } j \succsim_{D} i .
\end{aligned}
$$

It is well known that $\succsim_{D}$ is a preordering. The relation $\succsim_{D}$ (resp., $\left.\succ_{D}\right)$ is called the desirability (resp., strict desirability) relation, and $\approx_{D}$ is the equi-desirability relation.

Definition 2. Linear game ${ }^{2}$

A simple game $v$ is linear whenever the desirability relation $\succsim_{D}$ is complete.

Definition 3. The weak desirability relation ([3])

Let $v$ be a simple game and $i, j \in N$. Then

$$
\begin{aligned}
& i \succsim_{d} j \quad \Leftrightarrow \quad\left|\mathcal{C}_{i}(h)\right| \geq\left|\mathcal{C}_{j}(h)\right| \text { for any } h \text { with } 1 \leq h \leq n, \\
& i \succsim_{d} j \quad \Leftrightarrow \quad i \succsim_{d} j \text { and } j \succsim_{d} i, \\
& i \approx_{d} j \quad \Leftrightarrow \quad i \succsim_{d} j \quad \text { and } j \succsim_{d} i .
\end{aligned}
$$

Then $\succsim_{d}$ is a preordering called the weak desirability relation. The relation $\succ_{d}$ is the strict weak desirability relation and $\approx_{d}$ is the weak equi-desirability relation.

In [5] it is proved that the desirability relation is a sub-preordering of the weak desirability relation, that is to say, for any $i, j \in N, i \succsim_{D} j$ implies $i \succsim_{d} j$ and $i \succ_{D} j$ implies $i \succ_{d} j$.

Definition 4. Weakly linear game ([3])

A simple game $v$ is weakly linear whenever the weak desirability relation $\succsim_{d}$ is complete.

As stated in [3], the completeness of the desirability relation $\succsim_{D}$ implies the completeness of the weak desirability relation $\succsim_{d}$ so that all linear games are also weakly linear.

Moreover, if $v$ is a linear simple game then $v$ is weakly linear and the desirability relation $\succsim_{D}$ and the weak desirability relation $\succsim_{d}$ coincide.

\section{Other characterizations of the desirability and the weak desirability relations}

Given two different elements $i, j \in N$ we can establish a function

$$
\varphi_{j i}: \mathcal{P}_{j} \rightarrow \mathcal{P}_{i}
$$

\footnotetext{
$\overline{2}$ linear games are also called complete, ordered or directed games in the literature, see Taylor and Zwicker [16] for references on these names.
} 
defined by:

$$
\varphi_{j i}(S)= \begin{cases}S & \text { if } i \in S \\ (S \backslash\{j\}) \cup\{i\} & \text { if } i \notin S\end{cases}
$$

It is not difficult to see that $\varphi_{j i}$ is bijective and its inverse is $\varphi_{i j}$. Notice that, for any $S \in \mathcal{P}_{j}$ its image $\varphi_{j i}(S)$ always contain $S \backslash\{j\}$.

In the following subsections we study the restrictions of $\varphi_{j i}$ to $\mathcal{W}_{j}(h)$ and to $\mathcal{C}_{j}(h)$. We will see that we can characterize the relations $i \succsim_{D} j$ and $i \succsim_{d} j$ by using these restrictions.

\subsection{The restriction of $\varphi_{j i}$ to $\mathcal{W}_{j}(h)$}

It is clear that, for any $S \in \mathcal{W}_{j}(h), \varphi_{j i}(S) \in \mathcal{P}_{i}(h)$, but it is not always true (except for $h=n$ ) that $\varphi_{j i}(S) \in \mathcal{W}_{i}(h)$. The following proposition gives a characterization of this fact.

Proposition 1. Let $v$ be a simple game, $i$ and $j$ be different elements in $N$ and $h$ be an integer with $1 \leq h<n$. Then,

$$
\varphi_{j i}\left(\mathcal{W}_{j}(h)\right) \subseteq \mathcal{W}_{i}(h) \Leftrightarrow\left\{\begin{array}{l}
S \cup\{j\} \in \mathcal{W} \Rightarrow S \cup\{i\} \in \mathcal{W} \\
\text { for any } S \subseteq N \backslash\{i, j\} \text { with }|S|=h-1 .
\end{array}\right.
$$

\section{Proof:}

Assume that $\varphi_{j i}\left(\mathcal{W}_{j}(h)\right) \subseteq \mathcal{W}_{i}(h)$, and let $S \subseteq N \backslash\{i, j\}$ be such that $S \cup\{j\} \in \mathcal{W}$ and $|S|=h-1$. Taking $T=S \cup\{j\}$ it is $T \in \mathcal{W}_{j}(h)$ and, thus, $\varphi_{j i}(T) \in \mathcal{W}$ by hypothesis. But, since $i \notin T$, it is $\varphi_{j i}(T)=(T \backslash\{j\}) \cup\{i\}=S \cup\{i\}$. This proves that $S \cup\{i\} \in \mathcal{W}$.

Conversely, assume the hypothesis and let $S \in \mathcal{W}_{j}(h)$. To prove that $\varphi_{j i}(S) \in$ $\mathcal{W}_{i}(h)$ we only need to see that $\varphi_{j i}(S) \in \mathcal{W}$. There are two possibilities: a) If $i \in S$ then $\varphi_{j i}(S)=S$ and, obviously, $\varphi_{j i}(S) \in \mathcal{W}$. b) If $i \notin S$ then $\varphi_{j i}(S)=$ $(S \backslash\{j\}) \cup\{i\}$. Now, taking $T=S \backslash\{j\}$ it is $T \subseteq N \backslash\{i, j\},|T|=h-1$ and $T \cup\{j\}=S \in \mathcal{W}$, thus, $T \cup\{i\}=\varphi_{j i}(S) \in \mathcal{W}$.

Proposition 2. Let $v$ be a simple game, $i$ and $j$ be different elements in $N$. Then,

$$
\begin{aligned}
& i \succsim_{D} j \quad \Leftrightarrow \quad \varphi_{j i}\left(\mathcal{W}_{j}(h)\right) \subseteq \mathcal{W}_{i}(h) \text { for any integer } h \text { with } 1 \leq h \leq n \\
& i \approx_{D} j \quad \Leftrightarrow \quad \varphi_{j i}\left(\mathcal{W}_{j}(h)\right)=\mathcal{W}_{i}(h) \text { for any integer } h \text { with } 1 \leq h \leq n .
\end{aligned}
$$

\section{Proof:}

From Definition 1 it is $i \succsim_{D} j$ if and only if $S \cup\{j\} \in \mathcal{W} \Rightarrow S \cup\{i\} \in \mathcal{W}$ for any $S \subseteq N \backslash\{i, j\}$. Since $|S| \leq n-2$, from Proposition 1 this last assertion is satisfied if and only if $\varphi_{j i}\left(\mathcal{W}_{j}(h)\right) \subseteq \mathcal{W}_{i}(h)$ for any $h$ with $1 \leq h \leq n-1$. But it is always true that $\varphi_{j i}\left(\mathcal{W}_{j}(n)\right) \subseteq \mathcal{W}_{i}(n)$ and this proves the first part. The second part is an obvious consequence of the first one. 


\subsection{The restriction of $\varphi_{j i}$ to $\mathcal{C}_{j}(h)$}

Proposition 3. Let $v$ be a simple game, $i$ and $j$ be different elements in $N$ and $h$ be an integer with $1<h \leq n$. Then,

$$
\varphi_{j i}\left(\mathcal{C}_{j}(h)\right) \subseteq \mathcal{C}_{i}(h) \quad \Leftrightarrow \quad \begin{cases}\varphi_{j i}\left(\mathcal{W}_{j}(h)\right) & \subseteq \mathcal{W}_{i}(h) \\ \varphi_{j i}\left(\mathcal{W}_{j}(h-1)\right) \subseteq \mathcal{W}_{i}(h-1)\end{cases}
$$

\section{Proof:}

i) Assume that $\varphi_{j i}\left(\mathcal{C}_{j}(h)\right) \subseteq \mathcal{C}_{i}(h)$. To prove that $\varphi_{j i}\left(\mathcal{W}_{j}(h)\right) \subseteq \mathcal{W}_{i}(h)$ consider $S \in \mathcal{W}_{j}(h)$. If $S \backslash\{j\} \notin \mathcal{W}$ then $S \in \mathcal{C}_{j}(h)$ and, by the hypothesis, $\varphi_{j i}(S) \in$ $\mathcal{C}_{i}(h) \subseteq \mathcal{W}_{i}(h)$. Otherwise, if $S \backslash\{j\} \in \mathcal{W}$ then $\varphi_{j i}(S) \in \mathcal{W}$ because of the monotonicity of $v$ taking into account that $\varphi_{j i}(S) \supseteq S \backslash\{j\}$. Thus, in either case, $\varphi_{j i}(S) \in \mathcal{W}_{i}(h)$.

To prove that $\varphi_{j i}\left(\mathcal{W}_{j}(h-1)\right) \subseteq \mathcal{W}_{i}(h-1)$ consider $S \in \mathcal{W}_{j}(h-1)$. If $i \in S$ then, obviously, $\varphi_{j i}(S)=S \in \mathcal{W}$. Otherwise, if $i \notin S$ then $\varphi_{j i}(S)=(S \backslash\{j\}) \cup\{i\}$. If $\varphi_{j i}(S) \notin \mathcal{W}$ it would be $S \cup\{i\} \in \mathcal{C}_{j}(h)$ because $|S \cup\{i\}|=h-1, S \cup\{i\} \in$ $\mathcal{W}$, by monotonicity, and $(S \cup\{i\}) \backslash\{j\}=\varphi_{j i}(S) \notin \mathcal{W}$. Thus, by hypothesis, $\varphi_{j i}(S \cup\{i\}) \in \mathcal{C}_{i}(h)$. But, since $\varphi_{j i}(S \cup\{i\})=(S \cup\{i\})$, by monotonicity it would be $S \notin \mathcal{W}$ which is a contradiction. Thus, in either case, $\varphi_{j i}(S) \in \mathcal{W}_{i}(h-1)$.

ii) Conversely, assume that $\varphi_{j i}\left(\mathcal{W}_{j}(h)\right) \subseteq \mathcal{W}_{i}(h)$ and $\varphi_{j i}\left(\mathcal{W}_{j}(h-1)\right) \subseteq \mathcal{W}_{i}(h-1)$, and consider $S \in \mathcal{C}_{j}(h)$. To prove that $\varphi_{j i}(S) \in \mathcal{C}_{i}(h)$ we only need to prove that $\varphi_{j i}(S) \backslash\{i\} \notin \mathcal{W}$, because by the hypothesis $\varphi_{j i}(S) \in \mathcal{W}_{i}(h)$. If $i \notin S$ then $\varphi_{j i}(S)=(S \backslash\{j\}) \cup\{i\}$ and, thus, $\varphi_{j i}(S) \backslash\{i\}=S \backslash\{j\} \notin \mathcal{W}$ by the hypothesis. Otherwise, if $i \in S$ then $\varphi_{j i}(S)=S$. In this case, if $\varphi_{j i}(S) \backslash\{i\} \in \mathcal{W}$ it would be $\varphi_{j i}(S) \backslash\{i\} \in \mathcal{W}_{j}(h-1)$ because $\varphi_{j i}(S) \backslash\{i, j\} \notin \mathcal{W}$ (by monotonicity, since $\left(\varphi_{j i}(S) \backslash\{j\} \notin \mathcal{W}\right)$. Thus, by the hypothesis, $\varphi_{j i}(S)\left(\varphi_{j i}(S) \backslash\{i\}\right) \in \mathcal{W}_{i}(h-1)$. But $\varphi_{j i}(S)\left(\varphi_{j i}(S) \backslash\{i\}\right)=\varphi_{j i}(S) \backslash\{i\} \notin \mathcal{W}$, which is a contradiction.

Proposition 4. Let $v$ be a simple game, $i$ and $j$ be different elements in $N$ and $h$ be an integer with $1<h<n$. Then,

$$
\left.\begin{array}{l}
S \cup\{j\} \in \mathcal{W} \Rightarrow S \cup\{i\} \in \mathcal{W} \\
\text { for any } S \subseteq N \backslash\{i, j\} \text { with } h-2 \leq|S| \leq h-1
\end{array}\right\} \Leftrightarrow \varphi_{j i}\left(\mathcal{C}_{j}(h)\right) \subseteq \mathcal{C}_{i}(h) .
$$

Proof:

The property " $S \cup\{j\} \in \mathcal{W} \Rightarrow S \cup\{i\} \in \mathcal{W}$ for any $S \subseteq N \backslash\{i, j\}$ with $h-2 \leq$ $|S| \leq h-1 "$ is equivalent to " $\varphi_{j i}\left(\mathcal{W}_{j}(h)\right) \subseteq \mathcal{W}_{i}(h)$ and $\varphi_{j i}\left(\mathcal{W}_{j}(h-1)\right) \subseteq$ $\mathcal{W}_{i}(h-1)$ " from Proposition 1, and, from Proposition 3 , this is equivalent to $\varphi_{j i}\left(\mathcal{C}_{j}(h)\right) \subseteq \mathcal{C}_{i}(h)$.

Corollary 1. Let $v$ be a simple game, $i$ and $j$ be different elements in $N$ and $h$ be an integer with $1<h<n$. Then,

$$
\left.\begin{array}{l}
S \cup\{j\} \in \mathcal{W} \Rightarrow S \cup\{i\} \in \mathcal{W} \\
\text { for any } S \subseteq N \backslash\{i, j\} \text { with } h-2 \leq|S| \leq h-1
\end{array}\right\} \Rightarrow\left|\mathcal{C}_{j}(h)\right| \leq\left|\mathcal{C}_{i}(h)\right| .
$$


Corollary 2. Let $v$ be a simple game, $i$ and $j$ be different elements in $N$ and $h$ be an integer with $1<h<n$. Then,

$$
\left.\begin{array}{l}
\varphi_{j i}\left(\mathcal{C}_{j}(h-1)\right) \subseteq \mathcal{C}_{i}(h-1) \\
\varphi_{j i}\left(\mathcal{C}_{j}(h+1)\right) \subseteq \mathcal{C}_{i}(h+1)
\end{array}\right\} \Rightarrow \varphi_{j i}\left(\mathcal{C}_{j}(h)\right) \subseteq \mathcal{C}_{i}(h) .
$$

Proof:

From Proposition $4, \varphi_{j i}\left(\mathcal{C}_{j}(h-1)\right) \subseteq \mathcal{C}_{i}(h-1)$ implies that $S \cup\{j\} \in \mathcal{W} \Rightarrow$ $S \cup\{i\} \in \mathcal{W}$ for any $S \subseteq N \backslash\{i, j\}$ with $|S|=h-2$. Similarly, $\varphi_{j i}\left(\mathcal{C}_{j}(h+1)\right) \subseteq$ $\mathcal{C}_{i}(h+1)$ implies that $S \cup\{j\} \in \mathcal{W} \Rightarrow S \cup\{i\} \in \mathcal{W}$ for any $S \subseteq N \backslash\{i, j\}$ with $|S|=h-1$. And the two conditions together are equivalent to $\varphi_{j i}\left(\mathcal{C}_{j}(h)\right) \subseteq \mathcal{C}_{i}(h)$, again from Proposition 4.

Proposition 5. Let $v$ be a simple game, $i$ and $j$ be different elements in $N$. Then,

$$
\begin{aligned}
& i \succsim_{D} j \Leftrightarrow \varphi_{j i}\left(\mathcal{C}_{j}(h)\right) \subseteq \mathcal{C}_{i}(h) \text { for any integer } h \text { with } 1 \leq h \leq n \\
& i \approx_{D} j \Leftrightarrow \varphi_{j i}\left(\mathcal{C}_{j}(h)\right)=\mathcal{C}_{i}(h) \text { for any integer } h \text { with } 1 \leq h \leq n .
\end{aligned}
$$

\section{Proof:}

From Proposition 2 it is $i \succsim_{D} j$ if and only if $\varphi_{j i}\left(\mathcal{W}_{j}(h)\right) \subseteq \mathcal{W}_{i}(h)$ for any $h$ with $1 \leq h \leq n$. From Proposition 3, this is satisfied if and only if $\varphi_{j i}\left(\mathcal{C}_{j}(h)\right) \subseteq \mathcal{C}_{i}(h)$ for any $h$ with $1<h \leq n$. And, taking into account that $\mathcal{W}_{k}(1)=\mathcal{C}_{k}(1)$ for any $k \in N$, the first part is proved. The second part is an obvious consequence of the first one.

Proposition 6. Let $v$ be a simple game, $i$ and $j$ be different elements in N.Then the following assertions are equivalent:

(i) $\varphi_{j i}\left(\mathcal{C}_{j}(n)\right) \subseteq \mathcal{C}_{i}(n)$

(ii) $\left|\mathcal{C}_{j}(n)\right| \leq\left|\mathcal{C}_{i}(n)\right|$

(iii) $\varphi_{j i}\left(\mathcal{W}_{j}(n-1)\right) \subseteq \mathcal{W}_{i}(n-1)$

(iv) $N \backslash\{i\} \in \mathcal{W} \Rightarrow N \backslash\{j\} \in \mathcal{W}$

Proposition 7. Let $v$ be a simple game, $i$ and $j$ be different elements in $N$. Then the following assertions are equivalent:

(i) $\varphi_{j i}\left(\mathcal{C}_{j}(1)\right) \subseteq \mathcal{C}_{i}(1)$

(ii) $\left.\mid \mathcal{C}_{j}(1)\right)|\leq| \mathcal{C}_{i}(1) \mid$

(iii) $\{j\} \in \mathcal{W} \Rightarrow\{i\} \in \mathcal{W}$

\section{The $m$-desirability relations}

We are going to introduce a collection of new preorders in $N$. 
Definition 5. Let $v$ be a simple game and $i, j \in N$.

For any integer $m$ with $1 \leq m \leq n$ we define:

$$
\begin{aligned}
& i \succsim_{m} j \Leftrightarrow\left\{\begin{array}{l}
\left|\mathcal{C}_{j}(h)\right| \leq\left|\mathcal{C}_{i}(h)\right| \quad \text { for any } h \text { with } m \leq h \leq n, \text { and } \\
\varphi_{j i}\left(\mathcal{C}_{j}(h)\right) \subseteq \mathcal{C}_{i}(h) \text { for any } h \text { with } 1 \leq h<m .
\end{array}\right. \\
& i \succ_{m} j \Leftrightarrow i \succsim_{m} j \text { and } j \succsim_{m} i, \\
& i \approx_{m} j \Leftrightarrow i \succsim_{m} j \text { and } j \succsim_{m} i .
\end{aligned}
$$

Then $\succsim_{m}$ is called the $m$-desirability relation. The relation $\succ_{m}$ is the strict $m-$ desirability relation and $\approx_{m}$ is the $m$-equi-desirability relation.

Notice that, using Proposition 4, the second part of the definition of the $m$-desirability relation can be reformulated in the following way:

Remark 1. For any $m$ with $1<m \leq n$ it is

$$
\left\{\begin{array}{l}
\varphi_{j i}\left(\mathcal{C}_{j}(h)\right) \subseteq \mathcal{C}_{i}(h) \\
\text { for any } h \text { with } 1 \leq h<m
\end{array}\right\} \Leftrightarrow\left\{\begin{array}{l}
S \cup\{j\} \in \mathcal{W} \Rightarrow S \cup\{i\} \in \mathcal{W} \\
\text { for any } S \subseteq N \backslash\{i, j\} \text { with }|S| \leq m-2
\end{array}\right\}
$$

The following proposition states that the desirability relation and the weak desirability relation are particular cases of m-desirability relations, and that different values of $m$ can give the same $m$-desirability relation.

Proposition 8. Let $v$ be a simple game and $i, j \in N$. Then,

$$
\begin{aligned}
& i \succsim_{d} j \quad \Leftrightarrow \quad i \succsim_{2} j \quad \Leftrightarrow \quad i \succsim_{1} j . \\
& i \succsim_{D} j \quad \Leftrightarrow \quad i \succsim_{n} j \quad \Leftrightarrow \quad i \succsim_{n-1} j .
\end{aligned}
$$

Proof:

From Definition 5, it is clear that $i \succsim_{1} j$ if and only if $\left|\mathcal{C}_{j}(h)\right| \leq\left|\mathcal{C}_{i}(h)\right|$ for any $h$ with $1 \leq h \leq n$ and, from Definition 5 , this is equivalent to $i \succsim_{d} j$. On the other hand, $i \succsim_{2} j$ if and only if $\left|\mathcal{C}_{j}(h)\right| \leq\left|\mathcal{C}_{i}(h)\right|$ for any $h$ with $2 \leq h \leq n$ and $\varphi_{j i}\left(\mathcal{C}_{j}(1)\right) \subseteq \mathcal{C}_{i}(1)$. But this last condition is equivalent to $\left|\mathcal{C}_{j}(1)\right| \leq\left|\mathcal{C}_{i}(1)\right|$ and, thus, $i \succsim_{2} j \Leftrightarrow i \succsim_{d} j$.

Similarly, from Definition $5, i \succsim_{n} j$ if and only if $\left|\mathcal{C}_{j}(n)\right| \leq\left|\mathcal{C}_{i}(n)\right|$ and $\varphi_{j i}\left(\mathcal{C}_{j}(h)\right) \subseteq \mathcal{C}_{i}(h)$ for any $h$ with $1 \leq h<n$. But it is clear that $\left|\mathcal{C}_{j}(n)\right| \leq\left|\mathcal{C}_{i}(n)\right|$ if and only if $\varphi_{j i}\left(\mathcal{C}_{j}(n)\right) \subseteq \mathcal{C}_{i}(n)$ and, using Proposition $5, i \succsim_{n} j \Leftrightarrow i \succsim_{D} j$. Finally, $i \succsim_{n-1} j$ if and only if $\left|\mathcal{C}_{j}(n)\right| \leq\left|\mathcal{C}_{i}(n)\right|,\left|\mathcal{C}_{j}(n-1)\right| \leq\left|\mathcal{C}_{i}(n-1)\right|$ and $\varphi_{j i}\left(\mathcal{C}_{j}(h)\right) \subseteq \mathcal{C}_{i}(h)$ for any $h$ with $1 \leq h<n-1$. From Proposition 5 it is obvious that $i \succsim_{D} j \Rightarrow i \succsim_{n-1} j$. Conversely, if $i \succsim_{n-1} j$, since $\left|\mathcal{C}_{j}(n)\right| \leq\left|\mathcal{C}_{i}(n)\right|$ is equivalent to $\varphi_{j i}\left(\mathcal{C}_{j}(n)\right) \subseteq \mathcal{C}_{i}(n)$, applying Corollary 2 for $h=n-1$ we get $\varphi_{j i}\left(\mathcal{C}_{j}(n-1)\right) \subseteq \mathcal{C}_{i}(n-1)$ and, thus, $i \succsim_{n-1} j \Rightarrow i \succsim_{D} j$

Proposition 9. Let $v$ be a simple game. Then, for any $m$ with $1 \leq m \leq n$, the $m$-desirability relation is a preorder in $N$. 


\section{Proof:}

It is already known that the desirability relation and the weak desirability relation are preorders, so that we can assume $2<m<n-1$. To prove that the $m$-desirability relation is transitive, let $i, j, k$ be different elements in $N$ such that $i \succsim_{m} j \succsim_{m} k$. It is clear that $\left|\mathcal{C}_{i}(h)\right| \geq\left|\mathcal{C}_{j}(h)\right| \geq\left|\mathcal{C}_{k}(h)\right|$ for any $h$ with $m \leq h \leq n$, and thus $\left|\mathcal{C}_{i}(h)\right| \geq\left|\mathcal{C}_{k}(h)\right|$ for any $h$ with $m \leq h \leq n$. To prove that $\varphi_{k i}\left(\mathcal{C}_{k}(h)\right) \subseteq \mathcal{C}_{i}(h)$ for any $h$ with $1 \leq h<m$ we will see, using Remark 1 , that $S \cup\{k\} \in \mathcal{W} \Rightarrow S \cup\{i\} \in \mathcal{W}$, for any $S \subseteq N \backslash\{i, k\}$ with $|S| \leq m-2$. Thus, suppose that $S \subseteq N \backslash\{i, k\}$ is such that $|\bar{S}| \leq m-2$ and $S \cup\{k\} \in \mathcal{W}$. There are two possibilities:

If $j \notin S$ it is $S \subseteq N \backslash\{j, k\}$ and, since $j \succsim_{m} k,|S| \leq m-2$ and $S \cup\{k\} \in \mathcal{W}$, we have $S \cup\{j\} \in \mathcal{W}$. But it is also true that $S \subseteq N \backslash\{i, j\}$ and, since $i \succsim_{m} j$, we have $S \cup\{i\} \in \mathcal{W}$.

If $j \in S$, let $S^{\prime}=S \backslash\{j\}$. Then $S^{\prime} \cup\{k\} \subseteq N \backslash\{i, j\}$ and $S^{\prime} \cup\{k\} \cup\{j\}=$ $S \cup\{k\} \in \mathcal{W}$. Since $i \succsim_{m} j$ and $\left|S^{\prime} \cup\{k\}\right|=|S| \leq m-2$, it is $S^{\prime} \cup\{k\} \cup\{i\} \in \mathcal{W}$. But $S^{\prime} \cup\{i\} \subseteq N \backslash\{j, k\}$ and, since $j \succsim_{m} k$ and $\left|S^{\prime} \cup\{i\}\right|=|S| \leq m-2$, we have $S^{\prime} \cup\{i\} \cup\{j\}=S \cup\{i\} \in \mathcal{W}$.

Proposition 10. Let $v$ be a simple game and $i, j \in N$. Let $m, p$ be two integers with $1<m<p<n$. Then,

$$
\begin{aligned}
& i \succsim_{p} j \quad \Rightarrow \quad i \succsim_{m} j \\
& i \succ_{p} j \quad \Rightarrow \quad i \succ_{m} j
\end{aligned}
$$

\section{Proof:}

Assume that $i \succsim_{p} j$. To prove that $i \succsim_{m} j$, we only need to see that $\left|\mathcal{C}_{i}(h)\right| \geq$ $\left|\mathcal{C}_{j}(h)\right|$ when $m \leq h<p$, because the other cases are immediate consequence of the hypothesis. But, since $\varphi_{j i}\left(\mathcal{C}_{j}(h)\right) \subseteq \mathcal{C}_{i}(h)$ for any $h$ with $1 \leq h<p$, in particular it is $\left|\mathcal{C}_{i}(h)\right| \geq\left|\mathcal{C}_{j}(h)\right|$ when $m \leq h<p$.

Assume now that $i \succ_{p} j$, that is to say, $i \succsim_{p} j$ and $j \swarrow_{p} i$, and we will prove that $j \succsim_{m} i$. The fact that $i \succ_{p} j$ includes two possibilities:

(a) There is some $h$ with $p \leq h \leq n$ such that $\left|\mathcal{C}_{i}(h)\right|>\left|\mathcal{C}_{j}(h)\right|$. Since $h \geq p$ implies $h \geq m$, it is clear in this case that $j \succsim_{m} i$.

(b) There is some $h$ with $1 \leq h<p$ such that $\varphi_{i j}\left(\mathcal{C}_{i}(h)\right) \nsubseteq \mathcal{C}_{j}(h)$. This fact directly proves that $j \succsim_{m} i$ if $h<m$. If $m \leq h<p$ we will see that $\left|\mathcal{C}_{j}(h)\right|<$ $\left|\mathcal{C}_{i}(h)\right|$ and this also proves that $j \succsim_{m} i$. In effect, notice that $\varphi_{j i}\left(\mathcal{C}_{j}(h)\right) \varsubsetneqq \mathcal{C}_{i}(h)$, because $i \succsim_{p} j$ implies $\varphi_{j i}\left(\mathcal{C}_{j}(h)\right) \subseteq \mathcal{C}_{i}(h)$ and if $\varphi_{j i}\left(\mathcal{C}_{j}(h)\right)=\mathcal{C}_{i}(h)$ it would be $\varphi_{i j}\left(\varphi_{j i}\left(\mathcal{C}_{j}(h)\right)\right)=\mathcal{C}_{j}(h)=\varphi_{i j}\left(\mathcal{C}_{i}(h)\right)$ in contradiction with the hypothesis. Thus, $\left|\varphi_{j i}\left(\mathcal{C}_{j}(h)\right)\right|=\left|\mathcal{C}_{j}(h)\right|<\left|\mathcal{C}_{i}(h)\right|$.

The former proposition shows that the $m$-desirability relations form a chain of preorders on the set $N$ of players. Taking into account Propositions 8 and 10 we can write, for any two elements $i, j \in N$ :

$i \succsim_{D} j \Leftrightarrow i \succsim_{n} j \Leftrightarrow i \succsim_{n-1} j \Rightarrow i \succsim_{n-2} j \Rightarrow \cdots \Rightarrow_{i} j \succsim_{3} j i \succsim_{2} j \Leftrightarrow i \succsim_{1} j \Leftrightarrow i \succsim_{d} j$ $i \succ_{D} j \Leftrightarrow i \succ_{n} j \Leftrightarrow i \succ_{n-1} j \Rightarrow i \succ_{n-2} j \Rightarrow \cdots \succ_{3} j \succ j \succ_{2} j \Leftrightarrow i \succ_{1} j \Leftrightarrow i \succ_{d} j$

Notice, in particular, that all $m$-desirability relations coincide for $n \leq 3$, and that for $n=4$ they coincide either with the desirability or with the weak- 
desirability relation. As a consequence of this fact the $m$-desirability relations only appear as new preorders for $n \geq 5$.

In the following example all the $m$-desirability relations are shown.

Example 1. Let $N=\{1,2,3,4,5,6,7,8,9\}$ and $\mathcal{W}^{m}=\{\{1,2\},\{3,4,5\},\{6,7,8,9\}\}$

$$
\begin{aligned}
& 1 \approx_{D} 2 \quad 3 \approx_{D} 4 \approx_{D} 5 \quad 6 \approx_{D} 7 \approx_{D} 8 \approx_{D} 9 \\
& 1 \approx_{7} 2 \quad 3 \approx_{7} 4 \approx_{7} 5 \quad 6 \approx_{7} 7 \approx_{7} 8 \approx_{7} 9 \\
& 1 \approx_{6} 2 \quad 3 \approx_{6} 4 \approx_{6} 5 \quad 6 \approx_{6} 7 \approx_{6} 8 \approx_{6} 9 \\
& 1 \approx_{5} 2 \quad 3 \approx_{5} 4 \approx_{5} 5 \quad 6 \approx_{5} 7 \approx_{5} \quad 8 \approx_{5} 9 \\
& 1 \approx_{4} 2 \quad 3 \approx_{4} 4 \approx_{4} 5 \succ_{4} 6 \approx_{4} 7 \approx_{4} 8 \approx_{4} 9 \\
& 1 \approx_{3} 2 \succ_{3} 3 \approx_{3} 4 \approx_{3} 5 \succ_{3} 6 \approx_{3} 7 \approx_{3} 8 \approx_{3} 9 \\
& 1 \approx_{d} 2 \succ_{d} 3 \approx_{d} 4 \approx_{d} 5 \succ_{d} 6 \approx_{d} 7 \approx_{d} 8 \approx_{d} 9
\end{aligned}
$$

For any integer $m$ with $1 \leq m \leq n$ we can define the concept of $m$-linear game:

\section{Definition 6. m-linear game}

A simple game $v$ is $m$-linear whenever the $m$-desirability relation $\succsim_{m}$ is complete. The set of $m$-linear simple games will be denoted by $\mathfrak{L}(m)$.

From the above results it is clear that $\mathfrak{L}(n)=\mathfrak{L}(n-1)$ coincides with the set of linear games, $\mathfrak{L}(1)=\mathfrak{L}(2)$ is the set of weakly linear games, and

$$
\mathfrak{L}(n)=\mathfrak{L}(n-1) \subseteq \mathfrak{L}(n-2) \subseteq \cdots \subseteq \mathfrak{L}(3) \subseteq \mathfrak{L}(2)=\mathfrak{L}(1) .
$$

Thus, a linear game belongs to $\mathfrak{L}(m)$ for all $m(1 \leq m \leq n)$, and for any weakly linear (but not linear) game $v$ there exist some $m_{0}\left(2 \leq m_{0}<n-1\right)$ such that $v \notin \mathfrak{L}(m)$ for any $m>m_{0}$ and $v \in \mathfrak{L}(m)$ for any $m \leq m_{0}$. In the game of Example 1 it is $n=9$ and $m_{0}=3$. In the next example we show a weakly linear game which does not belong to any other class of $m$-linear games (in this case it is $n=5$ and $\left.m_{0}=4\right)$.

Example 2. Let $N=\{1,2,3,4,5\}$ and let $v$ be the game defined by

$$
\mathcal{W}^{m}=\{\{1,2\},\{1,3\},\{2,4\}\} .
$$

This game is not linear (does not belong to $\mathfrak{L}(5)=\mathfrak{L}(4)$ ) because the desirability relation only gives:

$$
1 \succ_{D} 3 \text { and } 2 \succ_{D} 4 \succ_{D} 5 .
$$

Clearly, $v$ is weakly linear (it belongs to $\mathfrak{L}(1)=\mathfrak{L}(2)$ ) with

$$
1 \approx_{d} 2 \succ_{d} 3 \approx_{d} 4 \succ_{d} 5 .
$$

But this game it is not 3-linear (it does not belong to $\mathfrak{L}(3)$ ) because $\{1,3\} \in \mathcal{W}$ and $\{2,3\} \notin \mathcal{W}$ implies $2 \swarrow_{3} 1$, and, similarly, $\{2,4\} \in \mathcal{W}$ and $\{1,4\} \notin \mathcal{W}$ implies $1 \succsim_{3} 2$. 


\section{Conclusions}

In this paper, a chain of classes of simple games is defined. In all of them, the $\mathrm{PBC}$ and the SS indices rank players in the same way, i.e., they are ordinally equivalent. The smallest class in the chain is the class of linear games and the largest one is the class of weakly linear games. We think that these classes will have an interesting role in future works. We can mention two open questions: a) For $n \geq 6$ it is known that all possible hierarchies are achievable in weakly linear games [8] but not all of them are achievable in linear games. Which is the smallest class $\mathfrak{L}(m)$ such that all hierarchies are already achievable in this class? b) It is known that the Johnston index is ordinally equivalent to PBC and SS indices in linear games but not in weakly linear games. Which is the largest class $\mathfrak{L}(m)$ in which Johnston index is ordinally equivalent to PBC and SS indices?

\section{Acknowledgements}

This research was partially supported by grant MTM2015-66818-P(MINECO/FEDER) from the Spanish Ministry of Economy and Competitiveness (MINECO) and from the European Union (FEDER funds).

\section{References}

1. J.F. Banzhaf. Weighted voting doesn't work: a mathematical analysis. Rutgers Law Review, 19(2):317-343, 1965.

2. F. Carreras and J. Freixas. Some theoretical reasons for using regular semivalues. In H. De Swart, editor, Logic, Game Theory and Social Choice. Proceedings of the International Conference, LGS, pages 140-154, Tilburg, the Netherlands, 1999.

3. F. Carreras and J. Freixas. On ordinal equivalence of power measures given by regular semivalues. Mathematical Social Sciences, 55(2):221-234, 2008.

4. J.S. Coleman. Control of collectivities and the power of a collectivity to act. In B. Lieberman, editor, Social Choice, pages 269-300. Gordon and Breach. New York, USA, 1971.

5. L. Diffo Lambo and J. Moulen. Ordinal equivalence of power notions in voting games. Theory and Decision, 53(4):313-325, 2002.

6. D.S. Felsenthal and M. Machover. The Measurament of Voting Power: Theory and practice, problems and paradoxes. Cheltenham: Edward Elgar, 1998.

7. J. Freixas, D. Marciniak, and M. Pons. On the ordinal equivalence of the Johnston, Banzhaf and Shapley power indices. European Journal of Operational Research, 216(2):367-375, 2012.

8. J. Freixas and M. Pons. Hierarchies achievable in simple games. Theory and Decision, 68(4):393-404, 2010.

9. J. Freixas and W.S. Zwicker. Weighted voting, abstention, and multiple levels of approval. Social Choice and Welfare, 21 (3):399-431, 2003.

10. J.R. Isbell. A class of simple games. Duke Mathematics Journal, 25(3):423-439, 1958.

11. R.J. Johnston. On the measurement of power: Some reactions to Laver. Environment and Planning A, 10(8):907-914, 1978. 
12. L.S. Penrose. The elementary statistics of majority voting. Journal of the Royal Statistical Society, 109(1):53-57, 1946.

13. S. Roy. The Ordinal Equivalence of the Jhonston Index and the Established Notions of Power. In Econophisics and Economics of Games, Social Choices and Quantitative Techniques, pages 372-380. Nex Economics Windows, part II, 2010.

14. L.S. Shapley. A value for n-person games. In A.W. Tucker and H.W. Kuhn, editors, Contributions to the theory of games II, pages 307-317. Princeton University Press, Princeton, USA, 1953.

15. L.S. Shapley and M. Shubik. A method for evaluating the distribution of power in a committee system. American Political Science Review, 48(3):787-792, 1954.

16. A.D. Taylor and W.S. Zwicker. Simple games: desirability relations, trading, and pseudoweightings. Princeton University Press, New Jersey, USA, 1999. 